\title{
Die transhumanistische Utopie des Mind-Uploading und die Grenzen der technischen Manipulation menschlicher Subjektivität
}

Zusammenfassung: In der Geschichte der Menschheit spielte die Verwendung von Technik eine entscheidende Rolle. Mit ihrer Hilfe ist dem Menschen nicht nur eine immer größer werdende Beherrschung der Natur und damit eine erhebliche Erleichterung seiner Arbeitsbedingungen ermöglicht worden. Vielmehr dient Technik auch dazu, die Lebensvollzüge des Menschen in unterschiedlichsten Bereichen zu unterstützen und $\mathrm{zu}$ optimieren.

Gerade die Erfolge im Zusammenspiel von Mensch, Natur und Technik haben mit dem sogenannten Transhumanismus eine intellektuelle Bewegung beflügelt, die eine Radikalisierung der Interaktion von Mensch und Technik anstrebt. Dem Transhumanismus zufolge soll die Technik nicht nur zur Verbesserung der Arbeitsbedingungen oder $\mathrm{zu}$ therapeutischen Zwecken dienen, letztlich soll auch die Natur des Menschen selbst durch die Verschmelzung mit Technik verbessert bzw. transformiert werden. Dadurch werde es möglich, sowohl die physische Basis als auch die psychisch-kognitive Verfassung des Menschen durch verschiedene Techniken zu perfektionieren. Dazu gehöre auch die Möglichkeit der technischen Manipulation und Reproduktion des menschlichen Bewusstseins (Mind-Upload oder Mind-Cloning).

Ziel dieses Beitrags ist es, die im Zusammenhang mit der transhumanistischen Transformation des Bewusstseins vorausgesetzten metaphysischen Konzepte kritisch zu rekonstruieren und zu untersuchen und dabei die prinzipiellen Grenzen des transhumanistischen Projekts aufzuzeigen. Dabei wird ein Subjektivitätsbegriff skizziert, der sowohl der spezifischen Natur des Bewusstseins als auch den wissenschaftlich-technischen Erkenntnissen und der durch sie ermöglichten technischen Manipulation Rechnung tragen kann. 


\section{Technik und menschlicher Lebensvollzug ${ }^{1}$}

Der Einsatz von Technik hat die menschlichen Lebensvollzüge seit jeher geprägt und wesentlich dazu beigetragen, dass der Mensch seine Umwelt seinen Zielen und Zwecken gemäß gestalten konnte. Während lange Zeit die Beherrschung der äußeren Natur im Vordergrund stand und damit die Erleichterung menschlicher Arbeitsbedingungen, richtet sich die technische Manipulation zunehmend auch auf die psychophysische Verfasstheit des Menschen. Dabei stehen bislang therapeutische Maßnahmen im Vordergrund, denn durch die technische Anwendung eines Störungsbeseitigungswissens lassen sich im medizinischen Kontext Teilfunktionen der jeweiligen Lebensvollzüge ersetzen oder zumindest regulieren (vgl. Janich 2006, 93-95), wobei sich die technischen Eingriffe sowohl auf körperliche als auch auf psychisch-geistige Aspekte des menschlichen Lebens erstrecken können. So können z. B. durch sogenannte Exoprothesen amputierte Gliedmaßen ersetzt und durch Sensoren Druck, Temperatur und Vibration erkannt und durch ein elektronisches Regelsystem verarbeiten werden. Auch können durch den Einsatz neurokybernetischer Prothesen (NCP) bei Epilepsiepatienten durch elektrische Impulse epileptische Anfälle vermieden werden. ${ }^{2}$ In ähnlicher Weise kann die „tiefe Hirnstimulation“, bei der Elektroden in das Gehirn eingesetzt werden, durch die eine elektrische Stimulation bestimmter Hirnareale ermöglicht wird, zu einer Linderung von therapieresistenten Depressionen oder Zwangserkrankungen führen (vgl. Schläpfer 2014, S 135-136).

Diese Erfolge im Zusammenspiel von Mensch, Natur und Technik haben mit dem sogenannten Transhumanismus eine intellektuelle Bewegung beflügelt, die eine Radikalisierung der Interaktion von Mensch und Technik anstrebt. Dem Transhumanismus zufolge soll die Technik nicht nur zur Verbesserung der Arbeitsbedingungen oder zu therapeutischen Zwecken dienen; letztlich soll auch die Natur des Menschen selbst durch die Verschmelzung mit Technik verbessert bzw. transformiert werden. Dadurch werde es möglich, sowohl die physische Basis als auch die psychisch-kognitive Verfassung des Menschen durch verschiedene Techniken zu perfektionieren oder gar zu transzendieren (vgl. Sorgner 2016, 34-64).

Die Menschheit ist demzufolge also in der Lage, ihre Entwicklung durch technische Eingriffe selbst in die Hand zu nehmen und ihre begrenzt Natur zu

1 Teile dieses Beitrags basieren (erweitert und modifiziert) auf Darstellungen und Argumentationen meines Artikels „Zur Anthropologie des Transhumanismus“.

2 Vgl. zur Thematik der Neuroprothetik auch die Aufsätze von Tobias Sitter und Olivia Mitscherlich-Schönherr in diesem Band. 
überwinden. Diese Entwicklung soll in der Zukunft zu einer „durchgängig rationalen Zivilisation, frei von psychischen Leiden, gesellschaftlichen Konflikten und physischen Entbehrungen [...]“ (Coenen 2007, 269) führen. Dies beinhaltet nicht nur die Verlängerung der Lebens- und Gesundheitsspanne; die meisten Transhumanisten gehen davon aus, dass es zukünftig möglich sein wird, das menschliche Bewusstsein auf einen Computer zu transferieren, so dass dies $\mathrm{zu}$ einer „praktischen Unsterblichkeit“ führen werde (vgl. Sorgner 2017, 157-159).

Dass die Realisierung eines solchen Zustands zukünftig tatsächlich im Bereich des technisch Machbaren liegt, wird oft durch eine Extrapolation des aktuellen technisch-wissenschaftlichen Fortschritts begründet (vgl. Göcke 2018, $134 \mathrm{f}$.): Dem wissenschaftlichen Fortschritt sei keine prinzipielle Grenze gesetzt, weshalb die gesamte Wirklichkeit früher oder später durch die naturwissenschaftliche Analyse beschreibbar und durch die entsprechende Technik auch manipulierbar werde. Damit setzt aber die von den Transhumanisten angestrebte Kontrollierbarkeit, Optimierbarkeit und Erzeugbarkeit des Bewusstseins bestimmte metaphysische Annahmen voraus, die für die Erfolgsaussichten des transhumanistischen Projekts konstitutiv sind. Denn nur wenn Bewusstsein als mit den materiellen Strukturen identisch oder als Funktion dieser Strukturen aufgefasst wird, ließe sich die erforderliche Manipulation durch technische Eingriffe auch tatsächlich bewerkstelligen. Die angestrebten Verbesserungen und Transformationen ließen sich nur dann realisieren, wenn sich das menschliche Bewusstsein als eine Art Datenstruktur entpuppt, die auf verschiedener Hardware implementiert werden könnte. Diese Annahme setzt eine reduktiv-naturalistische Auffassung der Wirklichkeit voraus, gemäß der alles Wesentliche in der Welt naturwissenschaftlich erfasst werden und somit auf kausal-funktionale Strukturen zurückgeführt werden kann. Zwar gibt es in der aktuellen Debatte ein ganzes Spektrum von Positionen, in denen diese zugrundeliegende reduktionistische Intuition auf der konzeptionellen Ebene unterschiedlich konkretisiert wird. Gemeinsam ist diesen Positionen des reduktiv-naturalistischen Spektrums aber, dass sie auf zwei Grundannahmen basieren:

1. Alle Wirklichkeit ist die Folge eines „bloßen Verhaltens“, das rein (wirk-) kausal-funktional beschrieben werden kann.

2. Das Verhalten des Gesamtsystems bzw. „höhere“ Eigenschaften werden durch die kausalen Dispositionen der Einzelteile der fundamentaleren Ebene bestimmt (vgl. Cramm 2008, S. 44).

Wirklichkeit ist dieser Auffassung gemäß durchgehend und ausschließlich kausal-funktional und bottom-up strukturiert: Die ganze Wirklichkeit erscheint als Geflecht von hinreichenden Wirkursachen, wobei die fundamentalen physischen Strukturen alle anderen Phänomene vollständig bestimmen. Diese Verfasstheit 
würde auch erst die vom Transhumanismus vorausgesetzte technische Manipulierbarkeit der Wirklichkeit garantieren, die auf kausalem Bewirkungswissen beruht: Kennt man die grundlegenden Strukturen und ihre Gesetzmäßigkeiten, so versteht man auch die auf ihnen basierenden Phänomene und kann sie einer technischen Manipulation zugänglich machen.

Ziel dieses Beitrags ist es zu zeigen, dass sich die reduktionistische Auffassung der Wirklichkeit, die der transhumanistischen Utopie zugrunde liegt, nicht einfach aus einer erfolgreichen Beschreibung der Wirklichkeit durch die Naturwissenschaften ergibt und dass die zusätzlich benötigten metaphysischen Prämissen letztlich zu unüberwindbaren Schwierigkeiten führen, wenn wesentliche Qualitäten menschlicher Subjektivität damit adäquat beschrieben werden sollen. Zudem kann anhand einer Analyse der Wissenschafts- und Technikpraxis als eines rationalen und normativen Handlungszusammenhangs gezeigt werden, dass gerade hier Aspekte der menschlichen Subjektivität vorausgesetzt werden müssen, die sich einer reduktiv-naturalistischen Sichtweise und somit auch einer vollständigen technischen Manipulierbarkeit im transhumanistischen Sinne entziehen.

\section{Ziele und Motive des Transhumanismus}

Auch wenn sich Motive und Ideen des Transhumanismus nach der Auffassung einiger seiner Vertreter schon sehr früh in alten Mythen finden lassen, beginnt eine intensive Beschäftigung mit der Thematik der Verbesserung und Überwindung der menschlichen Natur, bedingt durch den rasanten naturwissenschaftlich-technischen Fortschritt, erst im 20. Jahrhundert. ${ }^{3}$ Als einer der Schlüsseltexte gilt in diesem Kontext der Essay „The World, the Flesh and the Devil“ des irischen Physikers John Desmond Bernal (vgl. Coenen 2007, 270). In ihm entwarf Bernal eine Utopie, in der die menschliche Gattung letztlich vom „mechanischen Menschen“ abgelöst werden sollte. Zeichneten sich zu Bernals Zeit erstmals Techniken $\mathrm{ab}$, mit denen man auf die (biologische) Natur des Menschen Einfluss nehmen konnte, so beflügelte die aufkommende Computertechnologie und Kybernetik die transhumanistische Fantasie (vgl. Coenen 2007, 270). Es dauerte aber noch einige Jahrzehnte, bis der Transhumanismus die Mainstreamdebatten erreichte und heute als satisfaktionsfähige Position in aktuellen Diskussionen gilt. Maßgeblich für diese Aufwertung sind die großen technologischen Erfolge in jüngster Ver-

$3 \mathrm{Zu}$ den kulturellen Quellen des Transhumanismus vgl. auch den Artikel von Oliver Müller in diesem Band. 
gangenheit, die die menschliche Lebenswelt nachhaltig geprägt haben und die transhumanistische Utopie als realistische Option erscheinen lassen: Kommunikation, gesellschaftliche Interaktion, Arbeits- und Produktionsschritte vollziehen sich oftmals bereits vollständig digitalisiert und eröffnen so in diesen Feldern neue und weitreichende Optionen für das menschliche Selbstverständnis und damit in der transhumanistischen Sicht auch weitgehende Selbstgestaltungspotentiale.

Auch der enorme Fortschritt in der Entwicklung von Künstlicher Intelligenz, die in manchen Bereichen bereits heute den kognitiven Fähigkeiten des Menschen weit überlegen ist und bereits den Beginn einer tiefgreifenden gesellschaftlichen Veränderung darstellt, die unsere menschliche Lebenswelt massiv und in noch nicht absehbarer Weise beeinflussen wird, trägt wesentlich zur transhumanistischen Hoffnung bei. ${ }^{4}$ Denn der Fortschritt in der KI-Forschung wirkt sich auch auf die fundamentalste Ebene unseres menschlichen Selbstverständnisses aus, wenn es um die Frage geht, welche Qualitäten als wesentliche Aspekte unserer menschlichen Natur zu gelten haben. Ausgangspunkt hierfür sind die von den KISystemen erbrachten kognitiven Leistungen, die scheinbar autonom durchgeführt werden und durch außenstehende Beobachter nicht mehr nachvollzogen werden können. Hier drängt sich geradezu die Frage auf, worin sich menschlicher Geist und Bewusstsein noch von der technischen Simulation unterscheiden. Die daran anschließende Verhältnisbestimmung, die sowohl in Fachdiskussionen als auch in der breiteren Öffentlichkeit vorgenommen wird, weist eine interessante und in Spannung stehende Doppelbewegung auf: Einerseits wird der Erfolg der KI-Systeme als Beleg dafür aufgefasst, dass es sich beim Mensch eben auch nur um eine, wenn auch biologische und lernfähige Input-Output-Maschine handle, denn wie sonst könnten kognitive Leistungen simuliert werden, die die des Menschen in manchen Bereichen übertreffen? Der sogenannten Computermetapher zufolge verhält sich die Software zur Hardware, wie sich der Geist zum Gehirn verhält. Andererseits werden nun teilweise den KI-Systemen in einem starken Sinn mentale Fähigkeiten und Qualitäten wie Erleben, Denken, Erkennen, Wollen usw. zugeschrieben, so dass es mittlerweile schon ernsthaft geführte Diskussionen über die Frage gibt, ob solche KI-Systeme nicht als Person angesehen und ihnen damit Menschenrechte zugesprochen werden müssten. ${ }^{5}$ Wenn also - so die Vertreter des Transhumanismus - schon bald KI-Systeme kognitive Fähigkeiten besitzen, die den menschlichen ebenbürtig oder sogar überlegen sind, warum sollte

4 Für eine Übersicht über die Grundkonzepte und Herausforderungen der KI-Forschung vgl. Lenzen 2018.

5 Vgl. hierzu auch den Aufsatz von Armin Grundwald in diesem Band. 
es also nicht auch möglich sein, das Erleben und Denken konkreter Personen irgendwann vollständig zu manipulieren oder zu simulieren?

Gerade diese Erfolge werden oft als Belege dafür angeführt, dass die vom Transhumanismus angestrebte Transformation des Menschen bei weiterem Fortschritt der Technik in absehbarer Zeit realisiert werden kann (vgl. z. B. Bostrom 2018, 193). Wie genau diese Transformation erreicht werden kann und was letztlich das Ziel dieser Entwicklung sein soll, wird in den verschiedenen Strömungen des Transhumanismus unterschiedlich beantwortet. Oftmals wird der Transhumanismus von seinen Vertretern als eine Art Übergangsstufe angesehen, in der Teile der Menschheit in ein posthumanes Stadium übergehen werden. In diesem soll durch die technische Verbesserung des Menschen oder durch die Erschaffung posthumaner Intelligenzen eine neue Spezies etabliert werden, die die menschliche Gattung ersetzen soll, wie es letztlich im sogenannten Posthumanismus postuliert wird (vgl. Coenen 2007, 268).

Der Begriff „transhuman“ gelangt erstmals durch die englische Dante-Übersetzung und -Rezeption in die englische Sprache und wird dort 1957 von dem britischen Biologen, Humanisten und Schriftsteller Julian Huxley in dem Sammelband „New Bottles for New Wine“ aufgegriffen (vgl. Loh 2019, 32). Dort findet sich auch ein kleiner Abschnitt, in dem schon wesentliche Aspekte des transhumanistischen Denkens zusammengefasst werden:

„The human species can, if it wishes, transcend itself - not just sporadically, an individual here in one way, an individual there in another way, but in its entirety, as humanity. We need a name for this belief. Perhaps transhumanism will serve: man remaining man, but transcending himself, by realizing new possibilities of and for his human nature.“ (Huxley 1957, 17)

Wie bereits angedeutet, dauerte es noch Jahrzehnte, bis aus einer Intuition eine systematisch ausgearbeitete Position geworden ist. Dabei ist es auch unter Transhumanisten strittig, inwieweit sich die transhumanistischen Grundideen an den Humanismus zurückbinden lassen. Während manche seiner Vertreter wie Simon Young oder Nick Bostrom den Humanismus als Grundlage für den Transhumanismus ansehen oder sogar so weit gehen zu sagen, dass der Transhumanismus der mit technologischen Mitteln verwirklichte Humanismus sei, grenzen andere Vertreter wie Stefan Lorenz Sorgner den Transhumanismus explizit vom Humanismus ab (vgl. Sorgner 2016, 81).

Fest steht jedenfalls, dass der heutige Transhumanismus keine einheitliche Bewegung darstellt, sondern vielmehr als Sammelbegriff für verschiedene Strömungen aufgefasst werden muss. Allerdings lassen sich doch einige gemeinsame Ziele ausfindig machen, die den meisten Varianten des Transhumanismus als 
Leitidee dienen. Hava Tirosh-Samuelson hat diese Ziele wie folgt auf den Punkt gebracht:

„Transhumanists do not speak in one voice, and the movement expresses a variety of impulses, which are often at odds with each other. Nonetheless, several themes are common to transhumanist discourse: the view of evolving human nature, the focus on biotechnological enhancement that will exceed ordinary human physical and cognitive traits, a preoccupation with human happiness that can be perpetuated indefinitely, a deep concern for longevity and radical life extension, and a technoutopia of human machine fusion that constitutes practical immortality.“ (Tirosh-Samuelson 2011, 29)

Im Kern geht es dem Transhumanismus also um eine technisch kontrollierte Steuerung der Evolution, mit der durch die technische Manipulation der menschlichen Natur eine ständige Verbesserung der menschlichen Fähigkeiten einhergehe. Diese Entwicklung mündet den meisten Vertretern des Transhumanismus zufolge in einer technisch hergestellten starken Verbesserung und Kontrollierbarkeit des Geistes sowie letztlich in der Unsterblichkeit des menschlichen Bewusstseins. Grundlegend für diese Vision ist die oben bereits erwähnte reduktiv-naturalistische Deutung der Wirklichkeit, denn nur dann ergäbe sich die Möglichkeit, das Bewusstsein durch technische Eingriffe zu manipulieren oder gar in Perfektion zu simulieren (vgl. Bostrom 2018, 192f.). Als eine Art Datenstruktur wäre es somit - so die Vorstellung der Transhumanisten - auf einen Roboter transferierbar oder könnte nahezu unbegrenzt im Cyberspace weiterexistieren, was im Transhumanismus als „Mind-Uploading“ bzw. „Mind-Cloning“ bezeichnet wird. Dabei gehen die meisten Transhumanisten nicht von einer „echten“ Unsterblichkeit aus, denn es ist aus physikalischen Gründen sehr wahrscheinlich, dass kosmologische Entwicklungen jedem Leben früher oder später ein Ende bereiten: entweder durch die unendliche Dichte einer Singularität in einem sogenannten Big Crunch am Ende des Universums oder durch den Endzustand eines kosmologischen thermodynamischen Gleichgewichts, den sogenannten Wärmetod, in dem maximale Entropie erreicht und Leben ebenfalls verunmöglicht würde. Beiden Szenarien lässt sich auch mit technischen Hilfsmitteln aus rein physikalischen Gründen nicht entkommen, so dass mit „Unsterblichkeit“ im Transhumanismus letztlich nur ein sehr langes Leben gemeint sein kann (vgl. Sorgner 2017, 157-159).

Aber auch in diesem „bescheideneren“ Fall setzt die transhumanistische Vision bestimmte metaphysische Konzepte darüber voraus, was Bewusstsein ist und wie es sich technisch manipulieren bzw. transformieren lässt. 


\section{Metaphysische Voraussetzungen des Transhumanismus}

Während die bisherigen Erfolge technischer Manipulation nur eine kausale Beeinflussbarkeit psychophysischer Lebensvollzüge voraussetzen, die mit verschiedenen nicht-reduktionistischen Positionen in der Leib-Seele-Debatte kompatibel sind, setzt der Transhumanismus bezüglich seiner ambitioniertesten Ziele im Bereich menschlicher Lebensvollzüge eine reduktiv-naturalistische Ontologie voraus. Allerdings lässt sich durch eine genauere Analyse dieser Begründungsstrategie zeigen, dass die Erfolge heutiger technischer Manipulationsmöglichkeiten des Bewusstseins, wie sie sich z. B. in psychopharmakologischen oder neurokybernetisch-prothetischen Eingriffen einstellen, als Begründung für die mögliche Realisierung der weitreichenden Ziele des Transhumanismus nicht hinreichend sind. Denn diese Erfolge zeigen zunächst nur, dass durch technische Beeinflussung (beispielsweise der tiefen Hirnstimulation) der menschlichen Psyche bestimmte Störungen behoben werden können. Die Behauptungen des Transhumanismus gehen aber weit über diese Annahme und ihre vergleichsweise bescheidene metaphysische Voraussetzung einer Wechselwirkung von Geist und Körper hinaus. Denn die Perfektionierung des menschlichen Bewusstseins oder seine Reproduktion im „Mind-Uploading“ bzw. „Mind-Cloning“ setzt voraus, dass das Bewusstsein eine Art Datenstruktur ist, die sich technisch herstellen und dann auf verschiedene Träger transferieren lässt (vgl. Sorgner 2019, 37-41). ${ }^{6}$ Dies würde voraussetzen, dass das Bewusstsein entweder identisch mit seinen neuronalen Grundlagen ist oder sich erschöpfend durch seine funktionale Struktur charakterisieren lässt. Gemäß dieser metaphysischen Zusatzannahme werden alle Dimensionen unseres Mensch- und Personseins als kausales Produkt der zugrundeliegenden materiellen Konstellationen aufgefasst. Damit tritt an die Stelle des Menschen als eines handelnden Subjekts mit seinen geistigen Fähigkeiten und seelischen Dispositionen ein vollständig von physikalischen oder physiologischen Gesetzen bestimmtes materielles Substrat. Der Mensch wäre somit nur der Anwendungsfall einer alle Wirklichkeit umfassenden Doktrin: Alles, was geschieht, ist letztlich auf eine physikalische oder physiologische Struktur zurückführbar oder zumindest durch diese vollständig festgelegt.

Diese Auffassung steht aber in Spannung mit unserem lebensweltlichen Selbstverständnis, nach dem der Mensch als Subjekt aufgefasst wird, das nicht

6 Einige Vertreter des Transhumanismus gehen sogar so weit zu behaupten, dass unsere gesamte Wirklichkeit schon eine Computersimulation ist (vgl. Bostrom 2018). 
allein durch rein wirkkausale Faktoren festgelegt ist, sondern zumindest prinzipiell dazu fähig ist, seine Handlungen an Gründen zu orientieren und somit seine Interaktion mit anderen Subjekten und den Umgang mit seiner Umwelt freiheitlich durch Ziele, Werte und Zwecke zu bestimmen. Diese Fähigkeit, sich zu physischen und psychischen Dispositionen noch einmal denkend verhalten zu können, gehört zur wesentlichen Bestimmung des Menschen, und nur in diesem Fall, wenn also menschliche Handlungen und Erkenntnisse nicht gänzlich durch sachfremde Faktoren bestimmt sind, ist der Mensch überhaupt $\mathrm{zu}$ rationalen Handlungen fähig.

Da nun aber die vom Transhumanismus angestrebte Optimierung und Transzendierung der menschlichen Natur auf der metaphysischen Hintergrundannahme des reduktiven Naturalismus beruhen, gilt es zunächst für eine kritische Diskussion der transhumanistischen Ansprüche im Folgenden einige unhintergehbare begriffliche Minimalbestimmungen menschlicher Subjektivität zu rekonstruieren. Erst dann kann überhaupt sinnvoll gefragt werden, ob diese Qualitäten mit den dem Transhumanismus zur Verfügung stehenden Mitteln der kausal-funktionalen Analyse vollständig und adäquat erfasst werden können, was die Voraussetzung dafür wäre, dass sie sich auch im transhumanistischen Sinn technisch kontrollieren und manipulieren lassen. Es wird sich zeigen, dass die grundlegenden Qualitäten menschlicher Subjektivität - das phänomenale Bewusstsein und das Denken - bestimmte Charakteristika aufweisen, die diesem anspruchsvollen Ziel des Transhumanismus entgegenstehen.

\subsection{Grundlegende Konzepte menschlicher Subjektivität}

Es ist zwar richtig, dass es unterschiedliche „Menschenbilder“ gibt, in denen sich auch die unterschiedlichen kulturellen Betrachtungsweisen des Menschen widerspiegeln (vgl. Beck 2013, S. 35-56). Es gibt aber gute Gründe für die Annahme, dass es doch so etwas wie unhintergehbare begriffliche Minimalbestimmungen des Menschseins und der menschlichen Subjektivität gibt, die nicht nur transkulturell tief verankert sind, sondern sich letztlich für unsere lebensweltliche Praxis als unverzichtbar erweisen. Gemäß diesen setzen wir uns immer schon als empfindende und denkende Lebewesen voraus, die - zumindest prinzipiell - zu rationalen Handlungen fähig sind. Damit erkennen wir prinzipiell an, dass wir in der Lage sind, unter Abwägung von Gründen bestimmte Dinge zu tun oder zu lassen. Nur wenn vorausgesetzt wird, dass dem Handelnden in einer konkreten Situation andere Handlungsmöglichkeiten wirklich offenstehen und er das Vermögen besitzt, Gründe, die für sein Handeln und Urteilen relevant sind, abzuwägen, kann er auch für seine Handlungen verantwortlich gemacht werden (vgl. 
Nida-Rümelin 2007, 152). Für diese Fähigkeit der Selbstbestimmung sind zwei Modi von Subjektivität - das phänomenale Bewusstsein (beispielsweise in Form von Wahrnehmung) und das Denken - ausschlaggebend.

Beim sogenannten phänomenalen Bewusstsein handelt es sich um den Grundmodus von Subjektivität, weil dieser allen erlebenden Lebewesen zugeschrieben wird. Dabei ist in der aktuellen Debatte betont worden, dass es nicht möglich ist, Bewusstsein durch fundamentalere Begriffe $\mathrm{zu}$ definieren (vgl. Chalmers 1996, 4). Dies lässt sich u.a. auch daran erkennen, dass für Bewusstseinsphänomene mangels hinreichend bestimmter Begriffe oft Metaphern wie z. B. „Innenseite eines Organismus“ verwendet werden, um zumindest die Aufmerksamkeit auf dasjenige Phänomen zu lenken, zu dem alle bewusstseinsfähigen Lebewesen schon unmittelbar einen Zugang besitzen. Nur durch die jeweilige Vertrautheit mit diesem Phänomen wird es überhaupt möglich, die gemeinte Qualität zu beschreiben. In diesem Sinn kann man sich einer begrifflichen Bestimmung mit Thomas Nagel wie folgt nähern:

\begin{abstract}
„But no matter how the form may vary, the fact that an organism has conscious experience at all means, basically, that there is something it is like to be that organism. There may be further implications about the form of the experience; there may even (though I doubt it) be implications about the behavior of the organism. But fundamentally an organism has conscious mental states if and only if there is something that it is like to be that organism something it is like for the organism.“ (Nagel 1974, 436)
\end{abstract}

Nagel beschreibt hier zwei Grundeigenschaften des Bewusstseins: Einmal die phänomenale Qualität des bewussten Erlebens, dass es sich für ein erlebendes Lebewesen also irgendwie anfühlt, in diesem Zustand zu sein. Dieser Aspekt wird der „What-it-is-like-ness“-Aspekt des Bewusstseins genannt und tritt immer nur mit einem anderen Charakteristikum zusammen auf, das Nagel ebenfalls nennt: Die Qualität der „What-it-is-like-ness“ ist immer schon für ein Subjekt, das erlebt. Oder anders ausgedrückt: Erleben ist durch phänomenale Qualitäten bestimmt (z. B. einen bestimmten Roteindruck), und diese zeichnen sich dadurch aus, dass sie nicht wie z. B. physikalische Eigenschaften einfach „an Dingen“ vorkommen, sondern dass sie nur als Qualitäten eines Erlebens in einem erlebenden Lebewesen auftreten, das als Subjekt fähig ist, dieses Erleben zu haben (vgl. Searle 2000, 561). Dabei werden bestimmte einzelne qualitative Aspekte des phänomenalen Bewusstseins „Quale“ bzw. im Plural „Qualia“ genannt (vgl. Searle 2000, 560). ${ }^{7}$ Dazu gehören beispielsweise visuelle und auditive Eindrücke, Geruchs- und

7 Chalmers nennt noch „experience“, „what it is like“, „subjective experience“ und „phenomenal“, die alle mehr oder weniger das Gleiche bezeichnen (vgl. Chalmers 1996, 6). 
Tasterfahrungen, Gefühle usw. (vgl. Chalmers 1996, 6-10). Auch die Wahrnehmung ist demnach eine bestimmte Art des phänomenalen Bewusstseins, nämlich die Erscheinungsweise, wie sich Dinge einem erlebenden Subjekt im Bewusstsein präsentieren.

Diese Bezogenheit der erlebenden Lebewesen auf die Welt im Modus des phänomenalen Bewusstseins ist auch eine grundlegende Voraussetzung für ein denkendes Subjekt, Erkenntnisse über die Welt zu generieren. Denn im Denken als einem spezifischeren Modus von Subjektivität werden durch Gedanken Sachverhalte der Wirklichkeit erkannt, die dem Subjekt durch das phänomenal Erlebte vermittelt sind, da das Erkennen der Welt sich letztlich auf eine Art der Wahrnehmung bezieht, die ihrerseits auf etwas Wirkliches bezogen ist. Gerade hierin zeigt sich die wesentliche Verschränkung von Subjektivität und Welt. Konkrete Subjektivität tritt immer verkörpert auf und besitzt somit einen wesentlichen Bezug zum Organismus, der seinerseits schon in Naturzusammenhängen steht (vgl. Cramer 1999, 41). Damit ist das Wahrgenommene zwar immer im Modus des phänomenalen Bewusstseins gegeben, aber dieses ist immer durch den Organismus bedingt. Dieser bestimmt also durch die Verkörperung des Subjekts die konkreten Inhalte des phänomenalen Bewusstseins und hat für die Lebensvollzüge des Lebewesens eine besondere Bedeutung. Der Organismus bedingt nicht nur alle Erlebnisqualitäten mit, er ist auch für die Interaktion des Lebewesens mit der Welt zentral, da das Lebewesen nur durch ihn auf die Welt einwirken kann.

Diese Bedingtheit ist aber, zumindest bei der denkerischen Thematisierung der wahrgenommen Inhalte, nicht als eine vollständige Festlegung im Sinne einer Konditionierung zu denken. Denn ein weiteres konstitutives Merkmal des menschlichen Denkens besteht darin, dass es sich nicht nur in bereits vorgegebenen Bahnen bewegen, sondern Gewohnheiten und damit verbundene normative Vorgaben noch einmal hinterfragen und deren Anwendung kritisch reflektieren kann. Damit erschöpft sich Denken nicht in einem reinen Regelfolgen, denn die Festlegung und die kritische Prüfung, ob bestimmte Regeln in einem konkreten Fall Anwendung finden sollen, ist selbst schon Bestandteil einer rationalen Reflexion im Denken. Damit verfügt menschliches Denken über einen gewissen Grad an Spontaneität, was sich dadurch ausdrückt, dass das denkende Subjekt prinzipiell in der Lage ist, Inhalt und Richtung seiner Gedankengänge frei zu bestimmen. ${ }^{8}$

8 Mit diesen beiden Grundmodi ist die Beschreibung menschlicher Subjektivität natürlich noch nicht erschöpft. Aber sie sind Bestandteil einer unhintergehbaren Minimalbestimmung menschlicher Subjektivität, auf die auch umfassendere Subjekttheorien letztlich nicht verzichten können. 


\subsection{Metaphysische Deutungen des menschlichen Bewusstseins in reduktiv-naturalistischer Perspektive}

Die transhumanistischen Ziele der vollständigen Kontrolle und Reproduzierbarkeit des menschlichen Bewusstseins in Form einer Perfektionierung oder gar Simulation setzen, wie bereits gezeigt, eine reduktiv-naturalistische Perspektive voraus. Denn nur wenn Bewusstsein als mit den materiellen Strukturen identisch oder als Funktion dieser Strukturen aufgefasst wird, ließe sich die erforderliche Manipulation durch technische Eingriffe auch tatsächlich bewerkstelligen. Dies bedeutet, dass sich die genannten Minimalbestimmungen menschlicher Subjektivität mit den Annahmen eines reduktiven Naturalismus adäquat fassen lassen müssen. Auch wenn reduktiv-naturalistische Positionen sich in ihren konkreten Formulierungen unterscheiden, gemeinsam ist ihnen die Auffassung, dass letztlich die fundamentalen physischen Strukturen alle anderen Ebenen durch physikalische bzw. physiologische Gesetze vollständig festlegen sollen. Damit wird alles, was es gibt, rein wirkkausal bottom-up beschrieben: Die fundamentalen physischen Ebenen bestimmen auch alle „höheren“ Phänomene. In der Debatte um den reduktiven Naturalismus wurden in den letzten Jahrzehnten mit den Positionen der Identitätstheorie, der starken Supervenienz und des metaphysischen Funktionalismus verschiedene Konzepte diskutiert, wie diese reduktiv-naturalistische Sicht metaphysisch konkreter zu denken ist. ${ }^{9}$ Im Folgenden werden diese Konzepte, die dementsprechend als metaphysische Bezugspunkte der transhumanistischen Agenda angesehen werden müssen, und die mit ihnen verbundenen Probleme kurz skizziert. ${ }^{10}$

\section{Identitätstheorie und starke Supervenienz}

Während die identitätstheoretischen Ansätze behaupten, dass mentale Qualitäten schlicht mit physischen Strukturen identisch sind, behaupten Ansätze sogenannter starker Supervenienz zwar keine Identität, wohl aber, dass mentale $\mathrm{Zu}$ stände durch die ihnen zugrundeliegenden physischen Strukturen vollständig bestimmt sind.

9 Letztlich würde auch das Konzept sogenannter schwacher Emergenz unter diese Aufzählung fallen. Da schwache Emergenz letztlich starke Supervenienz voraussetzt, wird sie hier nicht eigens behandelt.

10 Einen Überblick über die verschiedenen physikalistischen Varianten des reduktiven Naturalismus und deren philosophische Probleme bietet z. B. Kutschera 2009, 140-170. 
Es ist hier nicht möglich, aber auch nicht notwendig, die weit verzweigte Diskussion der verschiedenen Argumentationsstrategien gegen diese Positionen zu referieren. Vielmehr soll anhand eines Arguments deutlich werden, dass beide Positionen an dem Problem der genuinen mentalen Verursachung scheitern. Wenn diese aber für rationale Erkenntnis, besonders in bestimmten Bereichen wie den der Wissenschaftspraxis, notwendig vorausgesetzt werden muss, dann kann der reduktive Naturalismus nicht wahr sein. ${ }^{11}$

Ausgangspunkt für dieses Argument ist die reduktiv-naturalistische Grundannahme, dass die fundamentalen physischen Strukturen mit ihren Kausalkräften schon hinreichend sind, um alle anderen Zustände (seien diese mental oder physisch) vollständig zu bestimmen. Da angenommen wird, dass eine hinreichende Ursache ihre Wirkung vollständig festlegt und es keine systematische Überdetermination durch mehrere hinreichende Ursachen geben kann, können mentale Qualitäten wie Wünsche oder Überzeugungen selbst keinerlei kausale Kraft sui generis besitzen. Das hieße, das vom Subjekt gelenkte Erzeugen und Entwickeln eines gedanklichen Zusammenhangs nach logischen Vorgaben wäre nicht möglich, weil die physikalische bzw. physiologische Mikroebene durch ihre kausalen Dispositionen gemäß einer starken Supervenienz (oder einer in dieser Hinsicht logisch äquivalenten physikalistischen Konzeption wie die der Identitätstheorie) auch alle diese Aspekte hinreichend festlegt.

Der reduktiv-naturalistischen Lesart zufolge ruft beispielsweise das physische Ereignis $\mathrm{P}_{1}$ durch Supervenienz das mentale Ereignis $\mathrm{M}_{1}$ hervor, und gleichzeitig ist es (bedingt durch das Prinzip der kausalen Geschlossenheit der physischen Welt) die hinreichende Ursache für das nachfolgende physische Ereignis $P_{2} \cdot M_{1}$ kann - wenn man systematische Überdetermination ausschließt - keinen Einfluss auf $\mathrm{P}_{2}$ besitzen, was bedeutet, dass ein mentales Ereignis kein physisches verursachen kann. Da $\mathrm{P}_{2}$ aber auch als hinreichende Realisierungsbasis für das mentale Ereignis $M_{2}$ angesehen wird, kann $M_{1}$ noch nicht einmal $M_{2}$ verursachen, womit auch eine mentale Verursachung auf der Ebene mentaler Ereignisse ausgeschlossen wäre. ${ }^{12}$ Das hieße, das vom Subjekt gelenkte Erzeugen und Entwickeln eines gedanklichen Zusammenhangs nach logischen Vorgaben wäre nicht möglich, weil die physikalische Mikroebene durch ihre kausalen Dispositionen gemäß einer starken Supervenienz (oder der Identitätstheorie) auch alle diese

11 Vgl. für eine ausführliche Analyse in Müller 2013, 131-143.

12 Es sollte hier schon angemerkt werden, dass „mentale Verursachung“ nicht bedeuten muss, dass die Entwicklung und Hervorbringung von Gedanken nach dem Muster eines strikten Ursache-Wirkung-Schemas wie bei Billardkugeln gedacht werden muss oder kann. Das Exklusionsargument soll zeigen, dass überhaupt eine kausale Relevanz von mentalen Gehalten bei Supervenienzphysikalismen nicht mehr gedacht werden kann. 
Aspekte hinreichend festlegt. Eine solche reduktiv-naturalistische Lesart der mentalen Verursachung ist demnach inkompatibel mit Rationalitätsstandards wissenschaftlicher und lebensweltlicher Urteilspraxis, nach der das denkende Subjekt in der Lage ist, seine Gedanken selbstbestimmt in eine bestimmte Richtung zu lenken, und damit die Möglichkeit besitzt, gedankliche Zusammenhänge nach inhaltlichen und logischen Kriterien zu entwickeln.

Dies lässt sich kurz an einem wesentlichen Merkmal der Wissenschaftspraxis illustrieren, denn diese erweist sich als normativer Handlungszusammenhang, in dem normative Vorgaben erfüllt werden müssen, um überhaupt $\mathrm{zu}$ den erwünschten Ergebnissen zu kommen. Besonders deutlich zeigt sich diese Normativität in der Experimentalpraxis, die für alle empirischen Wissenschaften konstitutiv ist. Im Experiment werden künstlich die Bedingungen herbeigeführt, unter denen die zu untersuchenden Faktoren kausal isoliert werden. Erst durch diese kausale Isolation ist ein störungsfreier Wirkverlauf herstellbar, der durch eine wissenschaftliche Theorie beschrieben werden kann.

Damit entpuppt sich Wissenschaftspraxis als ein geplantes Handeln zur Herstellung von Zuständen und Verläufen, die ohne das menschliche zweckgerichtete Handeln gar nicht zustande gekommen wären (vgl. Janich 2009, 152ff.). Die gesetzesartige Beschreibung eines Naturablaufs ist also nur dann möglich, wenn dieser unter idealisierten Bedingungen abläuft, was wiederum nur durch eine zweckgerichtete Handlung im Experiment durch Laborbedingungen erreicht werden kann. Das bedeutet, um einen beobachtbaren wirkkausalen Zusammenhang im Experiment herzustellen, bedarf es rationaler Handlungen, die sich ihrerseits an normativen Vorgaben orientieren. Handlungen sind auf zweifache Weise normativ: Zum einen können sie ge- oder misslingen, das bedeutet, sie können richtig oder falsch vollzogen werden. Zum anderen können sie erfolgreich oder erfolglos sein, je nachdem, ob der jeweilige Handlungszweck erreicht oder verfehlt worden ist (vgl. Janich 2009, 19). Damit wird im Subjekt die Fähigkeit vorausgesetzt, sich durch Sachgründe (Fragestellungen, Rationalität usw.) zu einer Handlung zu bestimmen, die normativen Bestimmungen genügen muss, wie sie z. B. in dem Prinzip der methodischen Ordnung vorliegen (vgl. ausführlicher Janich 2014, 30-32; Janich 1997, 116 f.). Dieses Prinzip besagt, dass in der wissenschaftlichen Praxis, vor allem in Experimenten, Teilhandlungen in einer bestimmten, nicht vertauschbaren Reihenfolge vollzogen werden müssen, um zu einem Ergebnis zu kommen. Nur so lässt sich ein sinnvolles Treiben von Wissenschaft von einem laienhaften Herumdrücken an Messapparaturen unterscheiden. Wie oben dargelegt, lässt sich eine solche Fähigkeit reduktiv-naturalistisch nicht fassen, weil sich im Bild des reduktiven Naturalismus alles Geschehen nur als das Wirken blinder Kausalkräfte der elementarsten physischen 
Ebene darstellt. Die Rationalität der Wissenschaftspraxis widerspricht somit einem wesentlichen Merkmal der reduktiv-naturalistischen Wirklichkeitsdeutung.

\section{Funktionalismus}

Die metaphysische Alternative zu Identitätstheorie und starker Supervenienz bezüglich der technischen Manipulierbarkeit menschlichen Bewusstseins findet sich im sogenannten Funktionalismus. Dieser ist zwar prinzipiell kompatibel mit verschiedenen ontologischen Ansätzen in der Geist-Gehirn-Debatte (vgl. hierfür z. B. Cursiefen 2008, 11; Kim 1998, 125), insofern eine ontologische Reduktion auf physikalische Strukturen nicht als notwendig, sondern nur als möglich behauptet wird. Weil der Funktionalismus aber zudem beansprucht, ohne einen Rückgriff auf mentales Vokabular auszukommen, erfreut er sich auch innerhalb des reduktiv-naturalistischen Positionenspektrums großer Beliebtheit. ${ }^{13}$

Ausgangspunkt für seine Entwicklung war das Argument der sogenannten multiplen Realisierung, das besagt, dass dieselben mentalen Zustände, wie z. B. das Fühlen von Schmerzen, in verschiedenen Lebewesen physisch unterschiedlich realisiert werden können. ${ }^{14}$ Der Schmerz eines Menschen und der eines Oktopusses als phänomenale Qualität basieren auf sehr unterschiedlichen physiologischen Strukturen. In diesem Sinne sind gleiche mentale Zustände also physisch multipel realisierbar.

Im funktionalistischen Ansatz werden mentale Zustände deshalb nicht primär mit physischen Strukturen, sondern mit ihren kausalen Rollen identifiziert, wodurch auch eine gewisse Unabhängigkeit der Funktion von ihrer physischen Realisierung behauptet wird. Konkret wird ein funktionaler Zustand als die Menge aller kausalen Relationen zwischen mentalen Zuständen und Umwelteinflüssen als Input und Verhaltensreaktionen als Output angesehen. Ned Block definiert Funktionalismus wie folgt:

„Functionalism is the doctrine that pain (for example) is identical to a certain functional state, a state definable in terms of its causal relations to inputs, outputs, and other mental states. The functional state with which pain would be identified might be partially characterized in terms of its tendency to be caused by tissue damage, by its tendency to cause the

13 Es gibt zwar eine prinzipielle Kompatibilität des Funktionalismus mit verschiedenen Positionen in der Geist-Gehirn-Debatte, aber die meisten Funktionalisten sehen eine physikalistische Ontologie als die erfolgversprechendste Version an. So kommt z. B. nach Kim im Grunde genommen nur die physikalische Realität als einzig vernünftige Realisierungsinstanz für die funktionalen Zusammenhänge in Frage (vgl. Kim 1998, 125).

14 Diese Argumentationslinie geht u. a. zurück auf Putnam 1975. 
desire to be rid of it, and by its tendency to produce action designed to shield the damaged part of the body from what is taken to cause it.“ (Block 1980, 257)

Diese starke Behauptung des sogenannten klassischen Funktionalismus hat sich aus verschiedenen Gründen als nicht haltbar erwiesen: So setzt z. B. schon die Bestimmung dessen, was als In- bzw. Output für einen spezifischen mentalen Zustand gelten kann, als gemeinsamen Bezugspunkt eine Bestimmung voraus, die nicht mehr durch funktionalistisches Vokabular beschrieben werden kann, denn anderenfalls wäre überhaupt nicht klar, auf was sich die jeweiligen In- bzw. Output-Konstellationen beziehen, warum also ganz verschiedene Input-OutputKonstellationen demselben mentalen Zustand zugerechnet werden sollen (vgl. Hoffmann 2013, 41). Darüber hinaus kann die funktionalistische Analyse den „What-it-is-like-ness"-Aspekt als das charakteristische Grundmerkmal des Bewusstseins nicht erklären, weil die Angabe der kausalen Rollen keine eindeutigen Rückschlüsse auf die jeweilige phänomenale Qualität zulässt. Damit ist das wesentliche Charakteristikum mentaler Zustände in funktionalistischen Ansätzen nicht mehr thematisierbar (vgl. Kim 1998, 126-128). Es ist zudem berechtigterweise daran gezweifelt worden, dass es für die meisten mentalen Zustände überhaupt eine eindeutige Charakterisierung durch kausale Rollen geben kann. Denn schließlich wissen wir aus Erfahrung, dass phänomenale Zustände bei verschiedenen Menschen, ja sogar bei derselben Person, von ganz unterschiedlichen Verhaltensweisen begleitet werden können, wenn sie sich überhaupt in einem Verhalten ausdrücken. So gibt es in der Anästhesie das bekannte Phänomen, dass es bei Operationen vorkommen kann, dass der Patient durch Muskelrelaxanzien komplett paralysiert ist und trotzdem alles - inklusive der Schmerzempfindung - bewusst erlebt, ohne dass sich dies körperlich eindeutig feststellen ließe (vgl. Domino et al. 1999, 1053-1061). Die skizzierten Schwierigkeiten machen schon deutlich, dass die Qualität des phänomenalen Erlebens und erst recht die des Denkens nicht identisch mit kausalen Rollen sein können, weil diese schlicht zu unbestimmt sind: Verhaltensweisen lassen keinen eindeutigen Rückschluss darauf zu, ob damit auch ein phänomenales Erleben oder ein bestimmter Denkakt verbunden ist, weil es keine eindeutige Verbindung zwischen beiden gibt. 


\section{Metaphysische Schwierigkeiten des Mind-Cloning bzw. Mind-Uploading}

Die vorangegangenen Überlegungen haben bereits deutlich gemacht, dass die fundamentalen theoretischen Annahmen einer reduktiv-naturalistischen Deutung des Bewusstseins, die bei vielen Zielen der transhumanistischen Agenda vorausgesetzt wird, mit erheblichen Schwierigkeiten konfrontiert sind. Damit fehlt auch die theoretische Begründung für die Behauptung, dass sich menschliche Subjektivität vollständig im transhumanistischen Sinne manipulieren lasse. Nachfolgend soll gezeigt werden, dass die transhumanistischen Konzepte des Mind-Uploading und Mind-Cloning starke metaphysische Zusatzannahmen voraussetzen, welche die Möglichkeit der technischen Reproduzierbarkeit des Bewusstseins als prinzipiell fragwürdig erscheinen lassen.

Vielen transhumanistischen Vertretern zufolge soll es zukünftig möglich sein, durch eine Simulation des jeweiligen Gehirns einer Person auch das dazugehörige Bewusstsein auf einem Computer zu erzeugen, es bei Bedarf zu vervielfachen oder auf einen geeigneten künstlichen Körper zu transferieren (vgl. Klaes 2018, 402). Wenn die konkreten Verschaltungen und Funktionen des jeweiligen Gehirns auf einem Computer simuliert würden - so die Hoffnungen der meisten Transhumanisten -, dann bekäme man damit auch zugleich eine perfekte Kopie des menschlichen Bewusstseins mit all seinen Fähigkeiten, Erinnerungen und Charakterzügen. Das Bewusstsein wird dementsprechend als eine Art Datenstruktur aufgefasst, was die Voraussetzung der angestrebten technischen Manipulation darstellt.

Einer Computerstruktur phänomenales Erleben und Denken zuzusprechen heißt nun zu behaupten, dass sich diese Qualitäten als rein kausal-funktionale Strukturen erweisen und durch algorithmische Manipulation erzeugt werden können. Wie stehen nun die Erfolgsaussichten für ein solches Vorhaben? Wie bereits oben angedeutet, sehen Transhumanisten den Fortschritt der KI-Forschung als Basis für ihre Hoffnungen. Denn die von KI-Systemen erbrachten „kognitiven“ Leistungen scheinen die Unterscheidung von menschlichem Geist bzw. Bewusstsein und technischer Simulation zu nivellieren. Aus diesem Grund ist es erforderlich, die technischen Mittel zu rekonstruieren, die für dieses Projekt zur Verfügung stehen. Denn nur wenn diese hinreichend wären, um Bewusstsein wirklich herstellen bzw. kopieren zu können, wäre so etwas wie Mind-uploading oder Mind-Cloning überhaupt möglich. Es geht also darum, was Computer bzw. KI-Systeme aufgrund ihrer Struktur hinsichtlich der Bewusstseinsqualitäten leisten können. 
Bei dieser Rekonstruktion ist zunächst das Konzept der Turing-Maschine zentral, denn nach den heute gemeinhin akzeptierten Ausführungen des Mathematikers Alan Turing ist der Computer eine Maschine, die nach syntaktischen Regeln formale Symbole manipulieren kann und dadurch algorithmisch - also durch schrittweise Umformung von Zeichenketten nach einem bestimmten sich wiederholenden Schema - Rechenschritte abarbeitet. Dies gilt ebenso für KISysteme, auch wenn diese durch Lernprozesse $\mathrm{zu}$ plastischen Input-OutputStrukturen führen und z. B. neue Datenmuster „erkennen“ können, die zuvor noch nicht in das System einprogrammiert waren (vgl. Mainzer 2010, 145-180). Durch diese Lernprozesse besitzt ein KI-System die Fähigkeit, eigenständig neue Korrelationen zwischen Datenmustern zu finden. Das führt in Fällen, in denen ein bestimmtes Datenmuster zu einer Problemlösung beiträgt, $\mathrm{zu}$ den besagten „kognitiven“ Fähigkeiten von KI-Systemen. Ist damit aber schon eine geistige Dimension dieser Systeme gegeben?

Wenn den Transhumanisten zufolge keine prinzipiellen, sondern nur graduelle Unterschiede zwischen diesen kognitiven Leistungen der KI-Systeme und dem menschlichen Geist bestehen, dann müssten sich die Fähigkeiten von Letzterem durch die technischen Mittel, die in der Struktur eines Computers verwendet werden, erschöpfend beschreiben lassen. Um beurteilen zu können, ob dies tatsächlich der Fall ist, sind zunächst verschiedene Ebenen in der Struktur des Computers zu unterscheiden, die im Zusammenhang mit der Zeichenverwendung stehen: 1. die physikalische Ebene des Computers, 2. die syntaktische Ebene (Regeln der Symbolmanipulation), 3. die semantische Ebene (Bedeutung der verwendeten Symbole). Für die Konzepte des Mind-Cloning bzw. des MindUploading müsste gezeigt werden, dass mit einer Kopie der physikalischen Ebene bzw. mit einer Simulation ihrer Funktionalität dann die beiden anderen Ebenen, die für die Zeichenverwendung und damit für geistige Akte konstitutiv sind, notwendig für den Computer mitgegeben sind, denn das geklonte Bewusstsein soll ja alle Eigenschaften des Originals allein durch die Eigenschaften der jeweiligen Datenstruktur erlangen. Durch eine Rekonstruktion der technischen Mittel und Voraussetzungen lässt sich aber zeigen, dass das Ineinandergreifen der verschiedenen Ebenen in der menschlichen Subjektivität durch ihre beiden Grundmodi - phänomenales Erleben und Denken - gewährleistet wird, während bei Computersystemen der Bezug der verschiedenen Ebenen künstlich von außen hergestellt wird.

Im Fall der menschlichen Subjektivität wird die semantische Dimension des Zeichengebrauchs durch die Verschränkung von phänomenalem Bewusstsein und Denken mit der Welt konstituiert. Dies lässt sich anhand des sogenannten semiotischen Dreiecks demonstrieren: Ein abstrakt verwendetes Zeichen hat nur dann Bedeutung, wenn es einen Interpreten hat (ein erlebendes und denkendes 
Subjekt), das das Zeichen einem Sachverhalt in der Welt zuordnet. Nur im Kontext eines qualitativen Weltbezugs können Zeichen etwas für einen Zeichennutzer repräsentieren. Diese semantische Dimension spielt demnach für geistige Tätigkeiten eine entscheidende Rolle. Wenn Denken nicht nur die syntaktische Manipulation von Symbolen ist, sondern einen aufgrund der semantischen Dimensionen der verwendeten Zeichen bedeutungsvollen Bezug zur Welt besitzt, der durch das phänomenale Bewusstsein, das Denken und deren Einbettungsverhältnisse grundgelegt ist, dann besteht für Vertreter des Mind-Uploading die Herausforderung darin zu zeigen, wie Computer als rein syntaktische Maschinen aus sich heraus diese qualitative Dimension des menschlichen Geistes besitzen bzw. erzeugen können.

Dass Computer die erforderlichen Qualitäten nicht besitzen, lässt sich anhand der Analyse der technisch investierten Mittel zeigen. Denn gemäß der reduktiven Auffassung des menschlichen Geistes, die dem Mind-Uploading zugrunde liegt, müsste sich die erforderliche Verschränkung von physikalischer Ebene, Syntax und Semantik allein durch die grundlegenden Eigenschaften ergeben (Syntax aus physikalischen Bestimmungen, semantische Bedeutung aus syntaktischen Regeln). Allerdings zeigt sich, dass sich dieser Übergang beim Computer von einer unteren Ebene (beginnend mit der physikalischen Ebene) zur nächst höheren nicht durch die inhaltlichen Bestimmungen der unteren Ebene ergibt. Hierfür sind zusätzliche Annahmen erforderlich, die nicht in der unteren Ebene enthalten sind. Das bedeutet, dass sowohl die syntaktische als auch die semantische Dimension „kognitiver“ Prozesse nicht im Computer selbst liegen, sondern durch den Programmierer bzw. Computernutzer ins Spiel kommen (vgl. Fischer 2003, 45).

So ergibt sich die Semantik eben nicht aus der Syntax, sondern ist das Ergebnis eines Abbildungsvorgangs. Wenn also der Computerfunktionalismus den menschlichen Geist als rein syntaktisch arbeitende Maschine auffasst, wird das Problem der Semantik einfach ausgeblendet. Die physischen Realisierungen der Datenstrukturen auf einem Computer haben also von sich aus keinen Bezug zu der semantischen Dimension, sie repräsentieren diese nur für einen externen Benutzer, der schon als erlebendes und denkendes Lebewesen vorausgesetzt werden muss.

Das bedeutet, dass man auch bei der Struktur eines KI-Systems, das menschliches Verhalten imitiert, von diesem nicht auf ein phänomenales Bewusstsein oder Denken schließen kann, denn - das hatte die Kritik am Funktionalismus deutlich gemacht - es besteht zwischen beiden keine notwendige und eindeutige Verbindung. Die kausal-funktionale Zuordnung von abstrakten Datenmustern - auch wenn sie in KI-Systemen durch das maschinelle Lernen und die damit verbundenen Trainingsdaten plastisch ist - erzeugt nicht die Qualität 
des „What-it-is-like-ness“ oder die des Für-das-Subjekt-Seins, die für Bewusstsein konstitutiv sind, weil sich diese Qualitäten einer rein kausal-funktionalen Charakterisierung entziehen. Das bedeutet umgekehrt auch, dass sie sich nicht durch kausal-funktionale Strukturen künstlich herstellen lassen, selbst wenn deren technische Gestaltung äußerst komplex sein mag.

Dies hat auch Konsequenzen für die Möglichkeit einer maschinellen Simulation des menschlichen Denkens, das einen Bezug zum phänomenalen Erleben immer schon voraussetzt. Denn nur durch das phänomenale Erleben besitzen die in den Gedanken verwendeten Begriffe einen qualitativen Weltbezug, der über eine rein formal bestimmte Semantik hinausgeht. Um Dinge qualitativ bestimmen zu können, genügt es nicht, dass einem abstrakten Muster ein anderes Muster zugeordnet wird, weil hierbei immer eine semantische Unterbestimmtheit bestehen bleibt, die aber in qualitativen Urteilen, die sich auf die konkrete Welt beziehen, notwendig aufgehoben sein muss. ${ }^{15}$

Zudem besteht ein weiterer wesentlicher Unterschied zwischen dem menschlichen Denken und seiner Computersimulation darin, dass menschliches Denken über die Fähigkeit verfügt, seine Gedankengänge frei zu bestimmen und damit auch die ihn leitenden normativen Vorgaben kritisch zu hinterfragen. Das denkende Subjekt ist prinzipiell in der Lage, eine beliebige Perspektive zu wählen, unter der ein Gegenstand thematisiert werden soll. Damit ist Denken nicht nur ein blindes Regelfolgen oder ein Folgen in durch Lernprozesse konditionierten Bahnen, sondern besitzt immer auch die Möglichkeit, Regeln, Normen und Kriterien, die festlegen, wann bestimmte Regeln angewandt werden sollen, kritisch zu diskutieren. Diese Fähigkeit ist aber prinzipiell nicht durch eine algorithmische Manipulation von Zeichenketten zu erreichen. Dies gilt, wie gesagt, auch für das maschinelle Lernen von KI-Systemen, deren Mustererkennung durch Trainingseinheiten zwar eine gewisse Plastizität besitzt, jedoch beruhen die dadurch gewonnenen Strukturen dennoch auf einer statistischen, rein kausalen und assoziativen Konditionierung und können somit nicht noch einmal auf einer Metaebene von dem System in Frage gestellt werden, so dass man KI-Systemen prinzipiell auch keine autonome Selbstbestimmung zuschreiben kann.

Selbst wenn es also gelänge, alle physikalischen Informationen über das Gehirn einer konkreten Person zu sammeln und auf einem Computer zu simulieren, wäre das nicht eine Kopie der Person, die über Erleben und selbstbestimmtes Denken verfügte. Dies folgt zum einen schon aus der Unterbestimmtheit

15 Ein ähnliches Problem ergibt sich in der Logik bei der sogenannten Extensionalitätsthese. Bestimmte intensionale Zusammenhänge lassen sich nicht allein aufgrund der extensionalen Verhältnisse darstellen (vgl. dazu Weingartner 1972, 127-178). 
der physikalischen Strukturen, die besagt, dass das phänomenale Erleben nicht durch kausal-funktionale Strukturen charakterisierbar ist und somit auch nicht künstlich hergestellt werden kann, zum anderen aus der Forderung, dass sich menschliches Denken prinzipiell zu seinen durch Gewöhnung gewonnen normativen Vorgaben noch einmal kritisch verhalten kann. ${ }^{16}$ Die Konzepte des MindCloning bzw. Mind-Uploading setzen aber genau diese kausal-funktionalistische Auffassung des Bewusstseins voraus, denn in der transhumanistischen $\mathrm{Zu}$ kunftsvision soll der menschliche Geist ja allein durch diejenigen Mittel dupliziert werden können, die in der Computertechnik prinzipiell zur Verfügung stehen.

\section{Ein kurzer Rückblick: Grenzen der technischen Manipulation menschlicher Subjektivität}

Die transhumanistischen Vertreter des Mind-Uploading bzw. Mind-Cloning verkennen somit den Abstraktheitsgrad der kausal-funktionalen Beschreibungsebene. Diese Beschreibungsebene hat zwar im Kontext der Gesamtbeschreibung des Lebewesens eine gewisse Berechtigung, insofern es sich eben um Beschreibungen von Teilaspekten handelt. Die konkrete Subjektivität von Lebewesen kann daher nicht ohne ihre Einbettungsverhältnisse gedacht werden. Das bedeutet auch, dass Subjektivität im Kontext des Lebendigen immer verkörpert auftritt und somit einen wesentlichen Bezug zum Organismus aufweist, der seinerseits schon in kausal-funktionale Naturzusammenhänge eingebettet ist. Diese Verschränkung von Subjektivität und Natur garantiert auch, dass Bewusstsein indirekt durch physische Kausalfaktoren beeinflussbar ist. Die Kritik an der reduktiv-naturalistischen Deutung menschlicher Subjektivität hat aber deutlich gemacht, dass sich eine Verabsolutierung der kausal-funktionalen Beschreibungsebene hinsichtlich des Bewusstseins nicht widerspruchsfrei denken lässt.

Damit muss aber ein umfassenderes Konzept des Menschen vorausgesetzt werden, in dem Erleben und Denken als nicht-reduzierbare Modi einer verkörperten Subjektivität aufgefasst werden. Dies führt keineswegs - wie wohl einige Vertreter des reduktionistischen Paradigmas befürchten - zu einer substanz-

16 Damit ist natürlich nicht gesagt, dass man bestimmte kognitive Aspekte des menschlichen Denkens nicht auf KI-Systeme auslagern könnte. Denn KI-Systeme sind über ihre trainierten künstlichen neuronalen Netzwerke gerade so konzipiert, dass sie sehr effektiv und ,eigenständig“ immer neue Datenmuster finden können, die dann Lösungen für bestehende Probleme darstellen, wobei sich die syntaktische und semantische Dimension aber letztlich durch Bezüge zum Programmierer und Benutzer ergibt. 
dualistischen Konzeption menschlicher Subjektivität. Die ontologische Minimalforderung, die sich aus der Kritik ergibt, lautet zunächst nur, dass die psychischgeistige Dimension nicht mit physiologischen Prozessen oder deren Funktionen identisch ist, eben weil damit wesentliche Qualitäten des Subjekts letztlich nicht mehr konsistent gedacht werden können. Es ist vielmehr nur verlangt, dass im Menschen das Psychisch-Geistige und das Physische als $\mathrm{zu}$ unterscheidende Momente eines Ganzen zu bestimmen sind, die in einem Bedingungsverhältnis zueinander stehen: Während das Psychisch-Geistige immer nur Moment eines lebendigen Lebewesens ist (und nicht etwa eine unabhängige Entität) und durch den Organismus mitbestimmt wird, ist umgekehrt der Organismus als eine besondere Art des Physischen, eben als Moment eines Lebewesens zu bestimmen, welches wiederum durch die psychisch-geistige Dimension mitbestimmt ist. Es geht also um zwei unaufhebbare Momente als Bestimmungen der Einheit eines Gesamtkomplexes - eben des Menschen als eines lebendigen Lebewesens. ${ }^{17}$ Die Verschränkung beider Dimensionen in einem lebendigen Lebewesen verbürgt damit auch - zumindest in einem bestimmten Umfang - eine indirekte technische Manipulierbarkeit der psychisch-geistigen Dimension. Die vorgetragene Kritik an einer reduktiv-naturalistischen Deutung menschlicher Subjektivität hat auch mit Blick auf das transhumanistische Projekt die Grenze der technischen Manipulation deutlich gemacht: Das weitreichende Ziel des Transhumanismus, Unsterblichkeit technisch realisieren zu können, ließe sich nur durch zusätzliche voraussetzungsreiche metaphysische Prämissen realisieren, die aber aus prinzipiellen Gründen dem Phänomen menschlicher Subjektivität nicht gerecht werden können.

\section{Literatur}

Beck, Birgit (2013): Ein neues Menschenbild? Der Anspruch der Neurowissenschaften auf Revision unseres Selbstverständnisses, Münster.

Bernal, John Desmond (1970): The World, the Flesh and the Devil: An Enquiry into the Future of the Three Enemies of the Rational Soul, London.

Block Ned (1980): „Are Absent Qualia Impossible?“, in: The Philosophical Review 89/2, 257274.

Bostrom, Nick (2018): Die Zukunft der Menschheit, Berlin.

Chalmers, David (1996): The Conscious Mind. In Search of a Fundamental Theory. New York / Oxford.

17 Für eine solche Konzeption vgl. z. B. Fuchs 2017. 
Coenen, Christopher (2007): „Transhumanismus“, in: Bohlken, Eike/Thies, Christian (Hg.): Handbuch Anthropologie. Der Mensch zwischen Natur, Kultur und Technik, Stuttgart, 268-276.

Cramer, Wolfgang ( $\left.{ }^{4} 1999\right)$ : Grundlegung einer Theorie des Geistes, Frankfurt am Main.

Cramm, Wolf-Jürgen (2008): „Zur kategorialen Differenz von Vernunft und Natur“, in: Cramm, Wolf-Jürgen/Keil, Geert (Hg.): Der Ort der Vernunft in einer natürlichen Welt. Logische und anthropologische Ortsbestimmungen, Weilerswist, 44-57.

Cursiefen, Stephan (2008): Putnam vs. Putnam. Für und wider den Funktionalismus in der Philosophie des Geistes, Hamburg.

Domino, Karen/Posner, Karen/Caplan, Robert/Cheney, Frederick (1999): „Awareness During Anesthesia: Closed Claims Analysis“, in: Anesthesiology 90/4, 1053-1061.

Fischer, Klaus (2003): „Drei Grundirrtümer der Maschinentheorie des Bewusstseins“, in: Köhler, Wolfgang R./Mutschler, Hans-Dieter (Hg.): Ist der Geist berechenbar?, Darmstadt.

Fuchs, Thomas (52017): Das Gehirn - ein Beziehungsorgan. Eine phänomenologisch-ökologische Konzeption, Stuttgart.

Göcke, Benedikt Paul (2018): „Designobjekt Mensch?! Ein Diskursbeitrag über die Probleme und Chancen transhumanistischer Menschenoptimierung“, in: Göcke, Benedikt Paul/Meier-Hamidi, Frank (Hg.): Designobjekt Mensch. Die Agenda des Transhumanismus auf dem Prüfstand, Freiburg, 117-151.

Grunwald, Armin: Technische Zukunft des Menschen? Eschatologische Erzählungen zur Digitalisierung und ihre Leitbilder, im vorliegenden Band.

Hoffmann, Thomas S. (2013): „Hermeneutischer Naturalismus“, in: Gerhard, Myriam/Zunke, Christine (Hg.): Die Natur denken, Würzburg, 27-55.

Huxley, Julian Sorell (1957): New Bottles for New Wine. Essays, London.

Janich, Peter (1997): Kleine Philosophie der Naturwissenschaften, München.

Janich, Peter (2006): „Der Streit der Welt- und Menschenbilder in der Hirnforschung“, in: Sturma, Dieter (Hg.): Philosophie und Neurowissenschaften, Frankfurt a. M., 75-96.

Janich, Peter (2009): Kein neues Menschenbild. Zur Sprache der Hirnforschung, Frankfurt. Janich, Peter (2014): Sprache und Methode. Eine Einführung in philosophische Reflexion, Tübingen.

Kim, Jaegwon (1998): Philosophie des Geistes, Wien / New York.

Klaes, Christian (2018): Was steckt hinter den Versprechen des Transhumanismus? Eine naturwissenschaftliche Perspektive, in: Göcke, Benedikt Paul/Meier-Hamidi, Frank (Hg.): Designobjekt Mensch. Die Agenda des Transhumanismus auf dem Prüfstand, Freiburg, 379-408.

Kutschera, Franz von (2009): Philosophie des Geistes, Paderborn.

Lenzen, Manuela (2018): Künstliche Intelligenz. Was sie kann \& was uns erwartet, München.

Loh, Janina (2019): Trans- und Posthumanismus zur Einführung, Hamburg.

Mainzer, Klaus (2010): Leben als Maschine? Von der Systembiologie zur Robotik und Künstlichen Intelligenz, Paderborn.

Mitscherlich-Schönherr, Olivia (2021): Ethisch-anthropologische Weichenstellung bei der Entwicklung von tiefer Hirnstimulation mit ,closed loop،, im vorliegenden Band.

Müller, Oliver (2021): Von der Selbstüberschreitung zur Selbstersetzung. Zu einigen anthropologischen Tiefenstrukturen des Transhumanismus, im vorliegenden Band.

Müller, Tobias (2013): „Zu Möglichkeit und Wirklichkeit mentaler Verursachung“, in: Philosophisches Jahrbuch 1/2013, 131-143. 
Müller, Tobias (2020): Zur Anthropologie des Transhumanismus, in: Watzka, Heinrich/Herzberg, Stephan (Hg.): Transhumanismus: Über die Grenzen technischer Selbstverbesserung, Berlin, 83-105.

Nagel, Thomas (1974): „What Is It Like to Be a Bat?“, in: The Philosophical Review 83/4, 435450.

Nida-Rümelin, Julian: „Freiheit als naturalistische Unterbestimmtheit“, in: Buchheim, Thomas/Pietrek, Torsten (Hg.): Freiheit auf Basis von Natur?, Paderborn (2007), 141-154.

Putnam, Hilary (1975): „The Nature of Mental States“, in: Putnam, Hilary (Hg.): Mind, Language and Reality. Philosophical Papers. Vol. 2, Cambridge, 429-440.

Schläpfer, Thomas et al. (2014): Tiefe Hirnstimulation in Neurologie und Psychiatrie, in: Der Nervenarzt (85) 2/2014, 135-136.

Searle, John (2000): „Consciousness“, in: Annual Review of Neuroscience 23, 557-578.

Sitter, Tobias (2021): Neurotechnologien aus der Perspektive einer Theorie konkreter Subjektivität, im vorliegenden Band.

Sorgner, Stefan Lorenz (2016): Transhumanismus ,Die gefährlichste Idee der Welt'!?, Freiburg.

Sorgner, Stefan Lorenz (2017): „Was wollen Transhumanisten?“, in: Göcke, Benedikt Paul/Meier-Hamidi, Frank (Hg.): Designobjekt Mensch. Die Agenda des Transhumanismus auf dem Prüfstand, Freiburg, 153-179.

Sorgner, Stefan Lorenz (2018): Schöner neuer Mensch, Berlin.

Tirosh-Samuelson, Hava (2011): „Engaging Transhumanism“, in: Hansell, Gregory R./Grassie, William (Hg.): Transhumanism and its Critics, Philadelphia, 19-54.

Weingartner, Paul (1972): „Die Fraglichkeit der Extensionalitätsthese und die Probleme der intensionalen Logik“, in: Haller, Rudolf (Hg.): Jenseits von Sein und Nichtsein, Graz, 127178. 